\title{
Dual inhibition of IGF1R and ER enhances response to trastuzumab in HER2 positive breast cancer cells
}

\author{
MARTINA S.J. MCDERMOTT ${ }^{1,5}$, ALEXANDRA CANONICI $^{1}$, LAURA IVERS $^{1}$, BRIGID C. BROWNE $^{1}$, \\ STEPHEN F. MADDEN ${ }^{2}$, NEILA. O'BRIEN ${ }^{3}$, JOHN CROWN ${ }^{1,4}$ and NORMA O'DONOVAN ${ }^{1}$ \\ ${ }^{1}$ National Institute for Cellular Biotechnology, Dublin City University, Dublin 9; ${ }^{2}$ Population Health \\ Sciences Division, Royal College of Surgeons in Ireland, Dublin 2, Ireland; ${ }^{3}$ Department of Medicine, \\ Division of Haematology/Oncology, David Geffen School of Medicine, UCLA, Los Angeles, CA 90095, \\ USA; ${ }^{4}$ Department of Medical Oncology, St Vincent's University Hospital, Dublin 4, Ireland
}

Received February 13, 2017; Accepted April 5, 2017

DOI: $10.3892 /$ ijo.2017.3976

\begin{abstract}
Although HER2 targeted therapies have improved prognosis for HER2 positive breast cancer, HER2 positive cancers which co-express ER have poorer response rates to standard HER2 targeted therapies, combined with chemotherapy, than HER2 positive/ER negative breast cancer. Administration of hormone therapy concurrently with chemotherapy and HER2 targeted therapy is generally not recommended. Using publically available gene expression datasets we found that high expression of IGF1R is associated with shorter diseasefree survival in patients whose tumors are ER positive and HER2 positive. IGF1R is frequently expressed in HER2 positive breast cancer and there is significant evidence for crosstalk between IGF1R and both HER2 and ER. Therefore, we evaluated the therapeutic potential of targeting ER and IGF1R in cell line models of HER2/ER/IGF1R positive breast cancer, using tamoxifen and two IGF1R targeted tyrosine kinase inhibitors (NVP-AEW541 and BMS-536924). Dual inhibition of ER and IGF1R enhanced growth inhibition in the four HER2 positive cell lines tested and caused an increase in cell cycle arrest in G1 in BT474 cells. In addition, combined treatment with trastuzumab, tamoxifen and either of the IGF1R TKIs enhanced response compared to dual targeting strategies in three of the four HER2 positive breast cancer cell lines tested. Furthermore, in a cell line model of trastuzumab-resistant HER2 positive breast cancer (BT474/Tr), tamoxifen combined
\end{abstract}

Correspondence to: Dr Norma O'Donovan, National Institute for Cellular Biotechnology, Dublin City University, Glasnevin, Dublin 9, Ireland

E-mail: norma.odonovan@dcu.ie

Present address: ${ }^{5}$ Department of Drug Discovery and Biomedical Sciences, South Carolina College of Pharmacy, University of South Carolina, Columbia, SC 29208, USA

Key words: erbB2, estrogen receptor, IGF1R, trastuzumab, tamoxifen with an IGF1R TKI produced a similar enhanced response as observed in the parental BT474 cells suggesting that this combination may overcome acquired trastuzumab resistance in this model. Combining ER and IGF1R targeting with HER2 targeted therapies may be an alternative to HER 2 targeted therapy and chemotherapy for patients with HER2/ER/IGF1R positive breast cancer.

\section{Introduction}

Of the $20-25 \%$ of breast cancers that are HER2 positive approximately 50-60\% also express estrogen receptor (ER) (1). Interaction between HER2 and ER is well documented since the observation by Benz et al that transfection of HER2 into ER positive cells results in tamoxifen resistance (2). It became widely accepted that HER2 overexpression causes intrinsic resistance to endocrine therapy and consequently HER2-targeted therapies combined with chemotherapy are recommended for patients with HER2 positive/ER positive breast cancer (1). However, response rates in these patients are lower than in HER2 positive/ER negative tumors (1). Furthermore, in HER2 positive/ER positive breast cancer patients, the benefit of trastuzumab progressively decreases as tumor expression of ER increases $(3,4)$. Thus, crosstalk between ER and HER 2 contributes to resistance to both endocrine therapy and HER2 targeted therapy. Studies of HER2 targeted therapies in combination with hormone therapy have shown clinical benefit in patients with HER2 positive/ER positive metastatic breast cancer $(5,6)$.

Crosstalk between ER and IGF1R signaling pathways stimulates proliferation of ER positive breast epithelial cells in vitro and in vivo $(7,8)$. The IGF1R ligands, IGF1 and IGF2, can activate un-liganded ER and have therefore been implicated in the regulation of ER activity. Estradiol (E2) can regulate IGF action by increasing IGF1R and insulin-receptor substrate expression in breast cancer cells resulting in an enhanced response to IGF1. IGF1 and E2 together synergistically stimulate proliferation of breast cancer cells (reviewed in ref. 9). Recent evidence also suggests that E2 and IGF1 can downregulate several potential tumor suppressors in breast cancer cells and negatively affect breast cancer outcome (10). 
This crosstalk between ER and IGF1R makes dual inhibition of the receptors an attractive target for the treatment of ER positive breast cancer and has been evaluated previously in preclinical models, whereby dual inhibition of ER and IGF1R results in enhanced apoptosis compared to single agent treatment (11-14). Anti-ER and anti-IGF1R targeted therapy has also been evaluated clinically in ER positive breast cancer patients who had progressed following first-line hormonal therapy (15). This study evaluated the addition of AMG-479, an anti-IGF1R monoclonal antibody to exemestane or fulvestrant (aromatase inhibitor/anti-estrogen) compared to single agent hormonal therapy. However, there was no significant increase in progression-free survival in the combination arm compared to single agent treatment. Interestingly, although this trial combined anti-ER and anti-IGF1R targeted therapy, IGF1R expression was not a selection criterion (15).

In HER 2 positive breast cancer, crosstalk between IGF1R and HER 2 has been extensively investigated in preclinical studies (16-19) and in a clinical trial. Despite promising preclinical data for combined HER2/IGF1R inhibition, the addition of the IGF1R targeted antibody cixutumumab to lapatinib plus capecitabine failed to show an improvement in progressionfree survival compared to lapatinib plus capetabine (20).

Previous work from our laboratory reported that phosphoIGF1R/IR staining was detected in $48.8 \%$ of HER2 positive breast tumors and in $44.7 \%$ of the HER2/ER positive breast tumors (19). While dual targeting of HER2 and ER or HER2 and IGF1R have been evaluated, combined targeting of ER and IGF1R together with HER2 targeted therapy has not been investigated in HER2 positive breast cancer. Therefore in the present study, we evaluated combined targeting of ER and IGF1R in HER2/ER/IGF1R positive breast cancer cell lines to determine its potential as a rational therapeutic strategy to improve treatment response in patients with HER2 positive ER positive tumors.

\section{Materials and methods}

Cell lines and culture reagents. Cell lines MCF7, BT474, MDA-MB-361 and HCC1419 cell lines were obtained from the American Type Culture Collection (Rockville, MD, USA). The EFM-192A cells were obtained from the German Tissue Repository DSMZ (Braunschweig, Germany). BT474/Tr cells were established by continuously culturing BT474 cells in $100 \mu \mathrm{g}$ of trastuzumab for a period of 9 months, as previously described (21). All cell lines except MDA-MB-361 were cultured in RPMI-1640 (Sigma-Aldrich, Wicklow, Ireland) supplemented with $10 \%$ fetal bovine serum (FBS) with $5 \%$ $\mathrm{CO}_{2}$. MDA-MB-361 cells were maintained in L-15 medium (Sigma-Aldrich) supplemented with $15 \% \mathrm{FBS}$, without $\mathrm{CO}_{2}$. All experiments were conducted with cells in the exponential growth phase and within 10 passages after thawing the cells. Cell line identity was authenticated by short tandem repeat (STR) typing (Source BioScience, Nottingham, UK). Stock solutions of BMS-536924 (Bristol Myers Squibb, Princeton, NJ, USA) and NVP-AEW541 (Novartis, Basel, Switzerland) (10 $\mathrm{mM})$ were prepared in dimethyl sulfoxide. Tamoxifen (Sigma-Aldrich) was dissolved in ethanol as a $10 \mathrm{mM}$ stock solution. Trastuzumab $(21 \mathrm{mg} / \mathrm{ml})$ was purchased from St Vincent's University Hospital (Dublin, Ireland).
Cell proliferation assays. Proliferation was measured using an acid phosphatase assay. Cells (3-5x10 $3 /$ well) were seeded in 96-well plates. Plates were incubated overnight at $37^{\circ} \mathrm{C}$, followed by addition of drug and incubation for 5 days. After washing with PBS, $10 \mathrm{mM}$ paranitrophenol phosphate substrate (Sigma-Aldrich) in $0.1 \mathrm{M}$ sodium acetate buffer with $0.1 \%$ Triton $\mathrm{X}-100$ (Sigma-Aldrich) was added to each well and incubated at $37^{\circ} \mathrm{C}$ for $2 \mathrm{~h}, 50 \mu \mathrm{l}$ of $1 \mathrm{M} \mathrm{NaOH}$ was added and the absorbance was read at $405 \mathrm{nM}$ (with reference wavelength $620 \mathrm{nM}$ ).

Cell cycle assay. Cell cycle assays were performed in 24-well plates with $2.5 \times 10^{4}$ cells/well. Following cell adherence, cell synchronization was achieved by replacing the growth media with serum-free RPMI for $16 \mathrm{~h}$. Cells were then drug treated at the indicated concentrations. After $48 \mathrm{~h}$ cells were trypsinized and transferred to a round-bottomed 96-well plate, washed with PBS and fixed with $70 \%$ ethanol overnight. Following fixation, the cells were washed with PBS and stained with Cell Cycle Reagent (Merck Millipore, Cork, Ireland), according to the manufacturer's protocol. Samples were measured on the Guava EasyCyte (Merck Millipore) and the data were analyzed using Modfit LT software (Verity Software House, Topsham, ME, USA).

Apoptosis assay. Apoptosis assays were performed in 24-well plates with $2.5 \times 10^{4}$ cells/well. After $24 \mathrm{~h}$ cells were drug treated at the indicated concentrations. After $72 \mathrm{~h}$ cells were trypsinized and transferred to a round-bottomed 96-well plate, washed with PBS and fixed with $70 \%$ ethanol at $4^{\circ} \mathrm{C}$ overnight. Following fixation the cells were washed with PBS and stained using the Guava TUNEL Assay kit (Merck Millipore), according to the protocol for the Guava EasyCyte (Merck Millipore). Apoptotic and non-apoptotic cell populations were determined and expressed as a percentage of the total cell population using the Guava TUNEL Software Module (Merck Millipore).

Immunoblotting. Whole cell lysates were prepared by seeding approximately $1 \times 10^{6}$ cells in $100-\mathrm{mm}$ cell culture petri dishes. Once the cells were $80 \%$ confluent the media was removed and the cell monolayer was washed twice with ice-cold PBS and then lysed with $500 \mu \mathrm{l}$ of RIPA buffer, containing $5 \mu \mathrm{l} 100 \mathrm{X}$ protease inhibitors, $5 \mu 1$ 100X PMSF and $5 \mu 1$ 100X sodium orthovanadate (Sigma-Aldrich). Cells were incubated on ice for $20 \mathrm{~min}$, sheared with a 21 -guage needle and centrifuged at $16,000 \mathrm{x} \mathrm{g}$ for $5 \mathrm{~min}$ at $4^{\circ} \mathrm{C}$. The supernatant was collected and protein concentration was determined using the BCA assay (Thermo Fisher Scientific, Hemel Hempstead, UK). Protein lysates $(50 \mu \mathrm{g})$ were resolved on $10 \%$ polyacrylamide gels (Lonza, Slough, UK), transferred to Hybond ECL nitrocellulose membrane (GE Healthcare, Cheshire, UK) and blocked using blocking solution [5\% milk powder (Bio-Rad,Hemel Hempstead, $\mathrm{UK}$ ) in $0.5 \%$ PBS-Tween] at room temperature for $1 \mathrm{~h}$ shaking. The membrane was incubated overnight, shaking, at $4^{\circ} \mathrm{C}$ with primary antibody: anti-ER $\alpha$ (Santa Cruz Biotechnology, Heidelberg, Germany) [1:200 in 3\% blocking solution (Bio-Rad)], anti-IGF1R $\beta$ (Santa Cruz Biotechnology) (1:666 in $5 \%$ blocking solution), anti-HER2 (Merck Millipore) (1:1000 in $5 \%$ blocking solution) and anti-tubulin (Sigma-Aldrich) (1:1000 

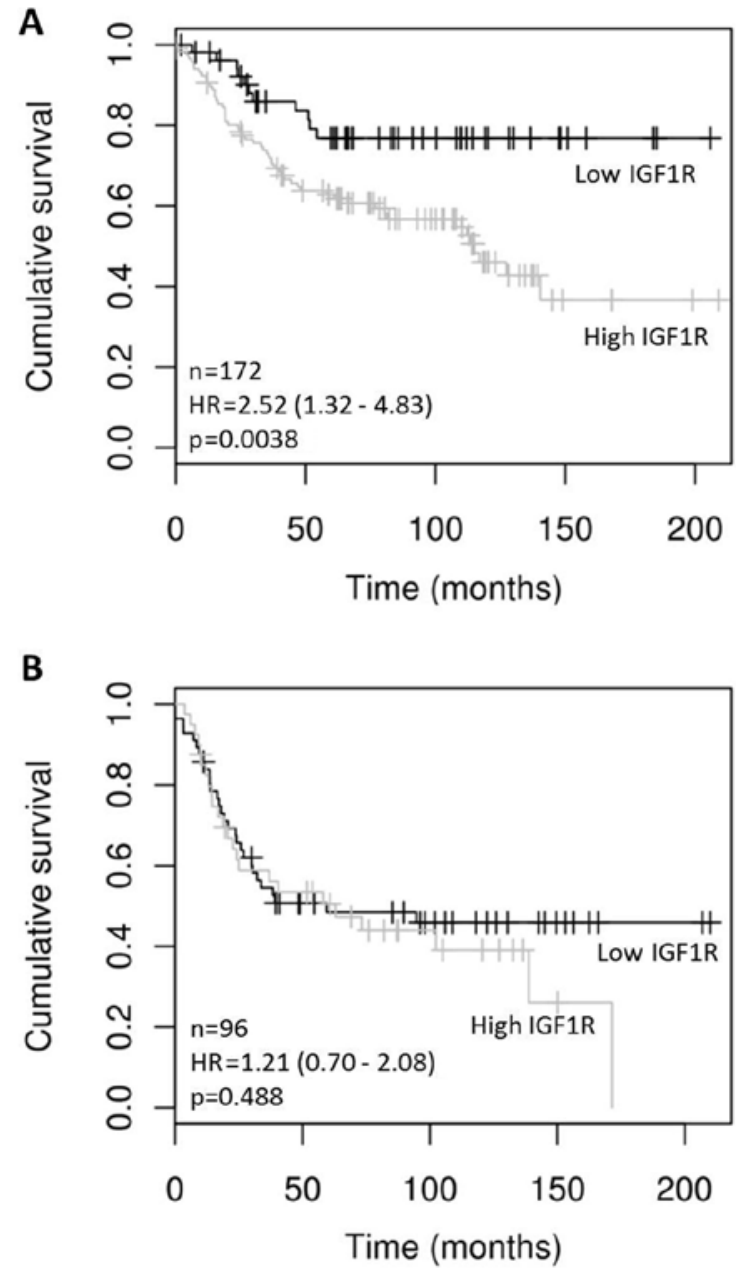

Figure 1. High expression of IGF1R is associated with decreased disease-free survival in HER2/ER positive breast cancer. IGF1R (entrez gene I.D. 3480) mRNA expression levels were stratified as low (lower than 25th percentile) or high (higher than the 25th percentile) in (A) ER positive and (B) ER negative breast tumors classified as HER2 subtype based on the gene classifier ssp2003 (23), using BreastMark (22). p-values and hazard ratios (HR) were generated using the log-rank test.

in 5\% blocking solution) or anti-GAPDH (R\&D Systems, Abingdon, UK) (1:1000 in $2.5 \%$ blocking solution). Three 10 min washes with $0.5 \%$ PBS-Tween were carried out followed by $1 \mathrm{~h}$ incubation with secondary antibody (antimouse (Sigma-Aldrich) or anti-rabbit [Thermo Fisher Scientific) (1:1000)], another three $10 \mathrm{~min}$ washes with $0.5 \%$ PBS-Tween and a PBS wash. Protein bands were detected using Luminol (Santa Cruz Biotechnology).

Enzyme-linked immunosorbant assay (ELISA). Total IGF1R was measured using a quantitative ELISA (R\&D Systems) according to the manufacturer's instructions. Lysates were prepared as described above and $50 \mu \mathrm{g}$ of protein was loaded per sample, in triplicate. Total IGF1R levels are presented as nanogram per milligram of total protein.

Gene expression analysis. BreastMark is an online algorithm which integrates gene expression and survival data from 26 datasets on 12 different microarray platforms corresponding to $\sim 17,000$ genes in up to 4,738 samples (22). Disease-free survival (DFS) and overall survival (OS) were analyzed and
Table I. Levels of IGF1R protein (ng/mg) measured by ELISA in five HER2 positive breast cancer cell lines.

\begin{tabular}{lc} 
Cell line & IGF1R $(\mathrm{ng} / \mathrm{mg})$ \\
\hline BT474 & $3.7 \pm 0.5$ \\
BT474/Tr & $3.1 \pm 0.4$ \\
EFM192-A & $0.6 \pm 0.1$ \\
MDA-MB-361 & $0.7 \pm 0.1$ \\
HCC1419 & $3.0 \pm 0.4$
\end{tabular}

the 25 th percentile was used to dichotomize IGF1R expression levels in ER positive breast tumors classified as HER2 subtype based on the gene classifier ssp2003 (23).

Statistical analysis. Analyses of the differences in response to treatment in in vitro assays were performed using the Student's t-test (two-tailed with equal variance). $\mathrm{p}<0.05$ was regarded as statistically significant. For the Kaplan Meier plots generated by BreastMark hazard ratios and $\mathrm{p}$-values were calculated using a log-rank test.

\section{Results}

IGFIR expression in HER $2 / E R$ positive tumors. Using publicly available gene expression data made accessible through BreastMark (22), we examined expression of IGF1R in tumors classified as HER2 positive using the ssp2003 subtype classifier (23). High expression of IGF1R (greater than the 25th percentile) correlated significantly with DFS in ER positive but not in ER negative HER2 positive tumors $(\mathrm{HR}=2.52$ (1.32-4.83), $\mathrm{p}=0.0038)$ (Fig. 1). IGF1R mRNA levels did not significantly correlate with OS in either HER2 positive ER positive $(\mathrm{p}=0.5054)$ or HER2 positive ER negative $(\mathrm{p}=0.0675)$ tumors.

Dual inhibition of IGF1R and ER. BT474 cells express ER and IGF1R and thus represent a cell line model of ER and IGF1R positive HER2 amplified breast cancer (Table I and Fig. 2). MCF7 cells, which are not HER2 amplified, were used as a positive control as they are a model of ER positive and IGF1R positive breast cancer wherein the two signaling systems engage in crosstalk resulting in synergistic growth (24). Tamoxifen inhibited the growth of both BT474 and MCF7 cells with an $\mathrm{IC}_{50}$ of $5.5 \pm 0.3 \mu \mathrm{M}$ and $5.7 \pm 0.5 \mu \mathrm{M}$, respectively, indicating that the cells display a similar sensitivity to ER inhibition (Fig. 2). IGF1R inhibition was examined with two anti-IGF1R TKIs, NVP-AEW541 (NVP) and BMS-536924 (BMS). MCF7 cells were equally sensitive to both NVP $(0.22 \pm 0.01 \mu \mathrm{M})$ and BMS $(0.19 \pm 0.02 \mu \mathrm{M})$, while BT474 cells, which express lower levels of IGF1R, were less sensitive to both NVP $(4.7 \pm 0.4 \mu \mathrm{M})$ and BMS (11.6 $\pm 1.1 \mu \mathrm{M})$ (Fig. 2).

To examine the effects of dual inhibition of ER and IGF1R, tamoxifen and NVP were administered as single agents and in combination at a fixed concentration. Despite the observation that BT474 cells were less sensitive to NVP as a single agent, the combined treatment with tamoxifen resulted in enhanced 



Figure 2. IGF1R inhibition in ER positive cells. (A) Detection of ER and HER2 in BT474, EFM-192A, MDA-MB-361 and HCC1419 cells. GAPDH was used as a control for protein loading. Growth of (B) MCF7 and (C) BT474 cells following 5-day treatment with tamoxifen, NVP-AEW541 (NVP) or BMS-536924 (BMS). Growth is expressed relative to control untreated cells and error bars represent the standard deviation of triplicate experiments.

A

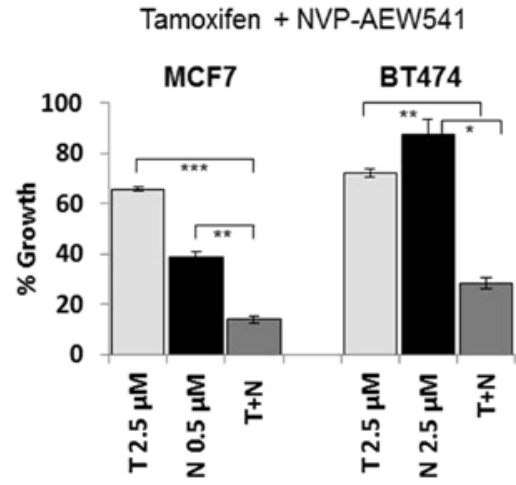

B

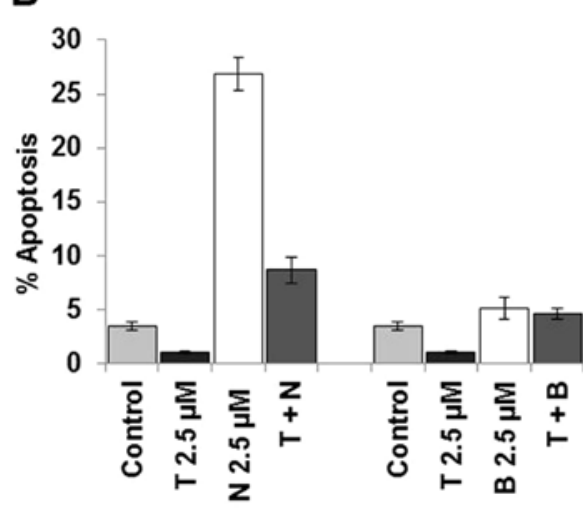

Tamoxifen + BMS-536924
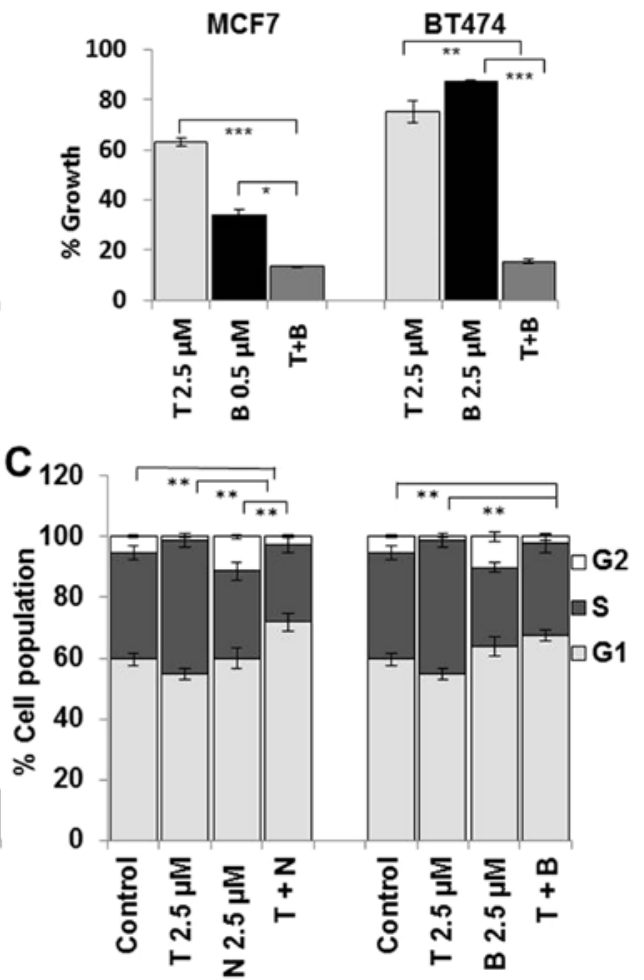

Figure 3. Dual targeting of IGF1R and ER inhibits proliferation of BT474 cells. (A) Growth of MCF7 and BT474 cells following 5-day treatment with the indicated concentrations of tamoxifen (T) and NVP (N) or BMS (B) alone and in combination. Growth is expressed relative to control untreated cells. (B) Percentage apoptosis induced in BT474 cells following $72 \mathrm{~h}$ treatment with the indicated concentrations of tamoxifen (T) and NVP (N) or BMS (B) alone and in combination. Apoptotic cell populations were identified using Guava TUNEL assay kit and expressed as a percentage of the total cell population using the Guava TUNEL Software Module. (C) Cell cycle analysis of BT474 cells following $72 \mathrm{~h}$ treatment with the indicated concentrations of tamoxifen (T) and NVP (N) or BMS (B) alone and in combination. Cells were stained using Cell Cycle Reagent (Merck Millipore) and the data was analyzed using Modfit LT software. Error bars represent standard deviation of triplicate experiments. p-values were calculated using Student's t-test where ${ }^{*} \mathrm{p} \leq 0.05,{ }^{* *} \mathrm{p} \leq 0.01$ and **** $\mathrm{p} \leq 0.001$. 

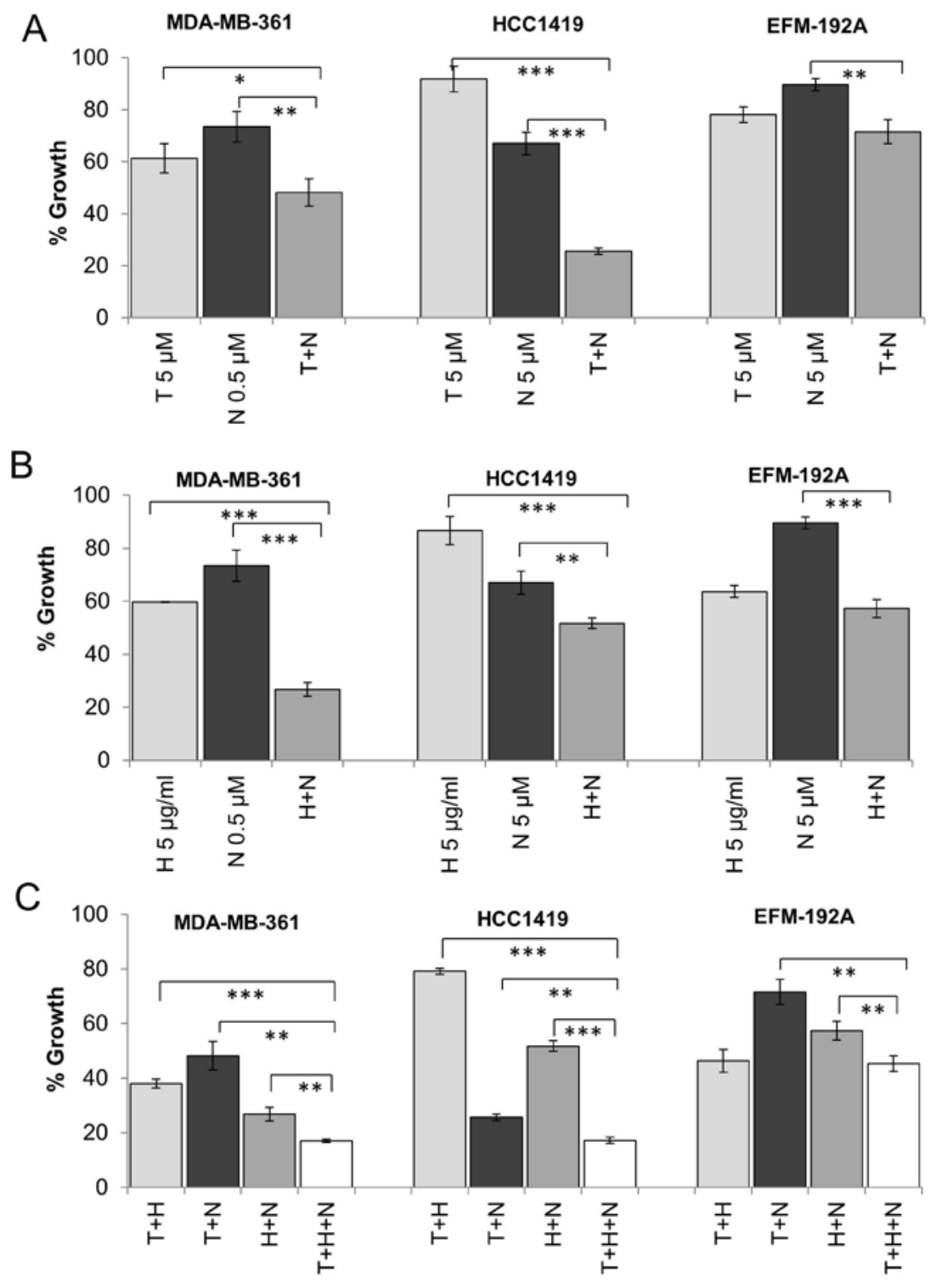

Figure 4. Triple therapy with tamoxifen, NVP and trastuzumab. Growth of MDA-MB-361, HCC1419 and EFM-192A cells following 5-day treatment with (A) tamoxifen (T) and NVP (N) alone and in combination, (B) trastuzumab $(\mathrm{H})$ and NVP $(\mathrm{N})$ alone and in combination and (C) tamoxifen, NVP and trastuzumab combined compared to dual combinations. Error bars represent standard deviation of triplicate experiments. p-values were calculated using Student's t-test where ${ }^{*} \mathrm{p} \leq 0.05,{ }^{* *} \mathrm{p} \leq 0.01$ and ${ }^{* * *} \mathrm{p} \leq 0.001$.

growth inhibition compared to tamoxifen alone $(\mathrm{p}<0.001)$ and NVP alone $(\mathrm{p}<0.001)$ (Fig. 3A). To confirm that the enhanced response to combined tamoxifen and NVP was due to targeting the ER and not tamoxifen off-target effects we also tested NVP in combination with fulvestrant and observed a similar enhanced response to the combination in BT474 cells (data not shown). The combination treatment also resulted in significantly greater growth inhibition in MCF7 cells compared to tamoxifen alone $(\mathrm{p}<0.0001)$ and compared to NVP alone $(p<0.001)$. Similar enhancement of growth inhibition was also seen when tamoxifen was combined with BMS in both cell lines (Fig. 3A).

Dual inhibition of ER and IGFIR results in G1 arrest. Single agent tamoxifen treatment did not induce apoptosis in BT474 cells, compared to control cells (Fig. 3B). Treatment with NVP alone induced significant apoptosis in BT474 $(\mathrm{p}<0.001)$ cells. NVP combined with tamoxifen did not increase apoptosis compared to treatment with NVP alone. BMS alone only induced a small increase in apoptosis in both cell lines and similar to NVP, when combined with tamoxifen did not induce a significant increase in apoptosis. These results suggest that the growth inhibitory effects of the combination treatment are not as a result of enhanced apoptosis. Neither tamoxifen nor NVP single agent treatment resulted in increased G1 accumulation (Fig. 3C). However, there was a significant increase in G1 accumulation following combined treatment compared to either tamoxifen alone $(\mathrm{p}=0.001)$ of NVP alone $(\mathrm{p}=0.010)$. A similar trend was also observed when BMS was combined with tamoxifen. These data suggest that the growth enhancement of the dual combination treatment is mediated by an increase in cell cycle arrest.

Dual ER and IGFIR inhibition in HER2 positive cell lines. Having established that dual targeting of ER and IGF1R results in greater inhibition of cell growth in BT474 cells, 

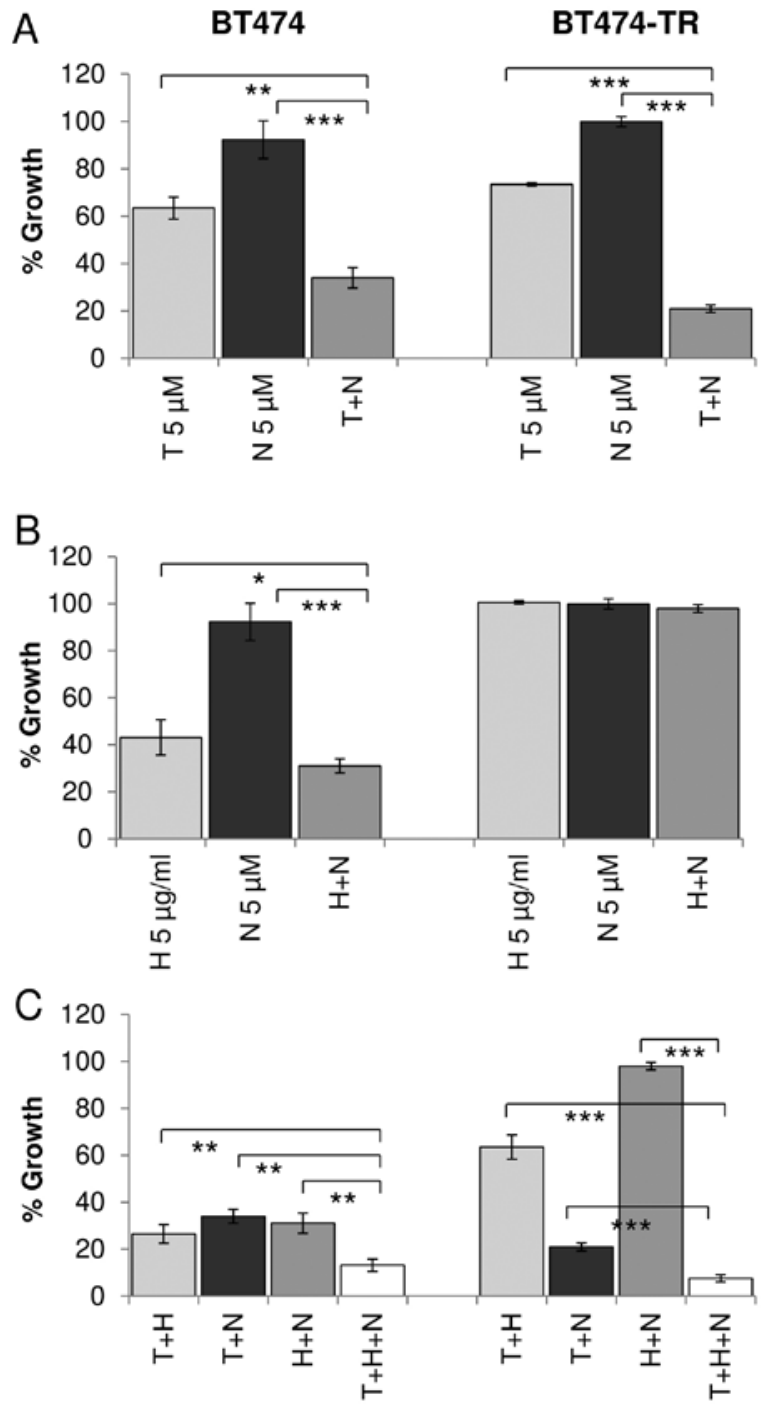

Figure 5. Triple therapy in trastuzumab resistant cells. Growth of BT474 and BT474/Tr cells following 5-day treatment with (A) tamoxifen (T) and NVP $(\mathrm{N})$ alone and in combination, $(\mathrm{B})$ trastuzumab $(\mathrm{H})$ and NVP $(\mathrm{N})$ alone and in combination and (C) tamoxifen, NVP and trastuzumab combined compared to dual combinations. Error bars represent standard deviation of triplicate experiments. p-values were calculated using Student's t-test where ${ }^{*} \mathrm{p} \leq 0.05,{ }^{* *} \mathrm{p} \leq 0.01$ and ${ }^{* * *} \mathrm{p} \leq 0.001$.

we tested the effects of dual therapy in three additional cell line models of HER2, ER and IGF1R positive breast cancer. MDA-MB-361, EFM-192A and HCC1419 cells are ER positive as determined by western blotting (Fig. 2) and expressed high levels of IGF1R determined by both western blotting and ELISA with EFM-192A cells exhibiting the lowest levels of IGF1R of the tested cell lines (Table I). MDA-MB-361 cells were more sensitive to single agent NVP treatment and thus were treated with a lower concentration of NVP compared to EFM-192A cells in these combination experiments. The combination of tamoxifen and NVP resulted in significantly greater inhibition of growth compared to tamoxifen or NVP alone in MDA-MB-361 ( $\mathrm{p}=0.041, \mathrm{p}=0.005$, respectively) and HCC1419 ( $\mathrm{p}<0.001, \mathrm{p}<0.001$, respectively) (Fig. 4A). An enhanced response was also observed for the combination in EFM-192A cells (28.5 $\pm 4.6 \%$ growth inhibition), although this did not achieve statistical significance compared to tamoxifen alone $(21.9 \pm 3.0 \%$ growth inhibition, $\mathrm{p}=0.106)$ (Fig. 4A). Similar results were also achieved when tamoxifen was combined with BMS (data not shown).

Dual targeting of IGFIR and HER2. We have shown that dual inhibition of IGF1R and ER is more effective than single agent treatment in four HER2/ER/IGF1R positive cell lines. We have previously shown that combined treatment with the HER2 targeted monoclonal antibody trastuzumab and IGF1R inhibitors produces enhanced response in some HER 2 positive breast cancer cell lines (19). Similarly, in this study, combined trastuzumab and NVP was more effective than trastuzumab or NVP alone in BT474 ( $\mathrm{p}=0.003, \mathrm{p}<0.001$, respectively), MDA-MB-361 ( $<<0.001, \mathrm{p}<0.001)$ and HCC1419 ( $<<0.001$, $\mathrm{p}=0.005$ ) (Figs. $4 \mathrm{~B}$ and $5 \mathrm{~B}$ ). The combination also produced greater growth inhibition than NVP $(\mathrm{p}<0.001)$ alone in the EFM-192A cells, but the effect of the combination (42.7 $\pm 3.4 \%$ growth inhibition) compared to trastuzumab alone $(36.3 \pm 2.3 \%$ growth inhibition) did not achieve statistical significance $(\mathrm{p}=0.055)$ (Fig. 4B). Similar results were also obtained when trastuzumab was combined with BMS in the three cell lines (data not shown).

Effect of triple targeted therapy on ER/HER2/IGF1R positive cells. Next we examined if there may be an additional benefit when trastuzumab, tamoxifen and IGF1R targeting are combined compared to dual inhibition. In three of the four cell lines tested (BT474, MDA-MB-361 and HCC1419), the triple combination was significantly more effective than dual inhibition with trastuzumab and NVP, tamoxifen and NVP or tamoxifen and trastuzumab (Figs. 4C and 5C). In the EFM-192A cells, the addition of NVP did not significantly enhance response compared to the double combination of tamoxifen and trastuzumab. Similar results were obtained for trastuzumab and tamoxifen were combined with BMS in the four cell lines (data not shown).

Effect of triple targeted therapy on trastuzumab-resistant cells. Trastuzumab-conditioned BT474/Tr cells were developed by continuous exposure to trastuzumab and represent a cell line model of acquired trastuzumab resistance which exhibits a significantly reduced response to trastuzumab compared to BT474 cells, as previously described (21). Similar to the parental BT474 cells, combined treatment with tamoxifen and NVP resulted in significantly greater inhibition of growth compared to either tamoxifen $(\mathrm{p}<0.001)$ or NVP $(\mathrm{p}<0.001)$ alone in the BT474/Tr cells (Fig. 5A). In BT474/Tr cells trastuzumab alone or in combination with NVP did not inhibit growth significantly (Fig. 5B). However, BT474/Tr cells were significantly more sensitive to the trastuzumab, tamoxifen and NVP triple combination compared to dual tamoxifen/NVP $(p<0.001)$, dual trastuzumab/NVP $(\mathrm{p}<0.001)$ and dual tamoxifen/trastuzumab $(\mathrm{p}<0.001)$ (Fig. 5C). Similar results were obtained with BMS in BT474/Tr cell line (data not shown).

\section{Discussion}

Based on the substantial evidence of crosstalk between ER and IGF1R signaling pathways, the inferior response rates to HER2-targeted therapies combined with chemotherapy for 
HER2/ER positive tumors, and previous studies from our laboratory suggesting that $45 \%$ of HER 2 positive/ER positive breast tumors are positive for phosphorylated IGF1R/IR (19), we tested combined targeting of ER and IGF1R in HER2 cell line models of HER2/ER/IGF1R positive breast cancer. Analysis of publicly available gene expression data provided further support for IGF1R playing a role in HER2 positive/ER positive breast cancer as higher expression of IGF1R mRNA is associated with shorter DFS whereas IGF1R expression did not correlate with DFS in the HER2 positive/ER negative cases.

Combined treatment with tamoxifen and the IGF1R inhibitor NVP was significantly more effective than single agent treatment in all cell lines tested. Combined tamoxifen and NVP treatment resulted in a significant increase in cells arrested in G1 phase without a significant increase in apoptosis. Two previous studies reported the enhanced growth inhibitory effects of combined ER/IGF1R targeted therapy corresponded with significant increases in apoptosis, but not cell cycle arrest $(12,13)$. The different effects noted may be due to the different cell culture conditions, different IGF1R targeted agents ( $\alpha$-IR-3, AG1024) (13) and/or different ER targeted agents (letrozole) (12) used. Furthermore, both NVP-AEW541 and BMS-536924 also target the insulin receptor $(25,26)$, thus inhibition of IR signaling may contribute to the induction of cell cycle arrest. Targeting the IR may also be beneficial in HER 2 positive breast cancer as IR signaling has been implicated in tumor progression in preclinical models of HER2 positive breast cancer (27). Of note, dual targeting of ER and IGF1R produced a similar level of growth inhibition in the trastuzumab resistant BT474 cells, as in the parental cells, suggesting that this ER/IGF1R targeting strategy may be beneficial in patients with HER2/ER/ IGF1R positive breast cancer following disease progression on trastuzumab-based treatment.

Similar to our previously published results (19), trastuzumab combined with NVP or BMS produced an enhanced response in the HER2 positive breast cancer cell lines tested. Targeting all three receptors (HER2, ER and IGF1R) simultaneously produced a significantly enhanced response compared to targeting two of the receptors in four of the five HER2 positive breast cancer cell lines tested, including the trastuzumab resistant BT474/Tr cell line. In EFM-192A cells, addition of NVP did not significantly improve the anti-proliferative effect compared to trastuzumab combined with tamoxifen. The EFM-192A cells have the lowest levels of IGF1R of the cell lines tested $(0.6 \pm 0.1 \mathrm{ng} / \mathrm{mg})$, although similar to MDA-MB-361 $(0.7 \pm 0.1 \mathrm{ng} / \mathrm{mg})$ (Table I). While IGF1R levels alone may not account for sensitivity or resistance to IGF1R inhibition, we have previously reported that high levels of IGF1R are weakly associated with greater sensitivity to NVP-AEW541 in a panel of 9 HER2 positive breast cancer cell lines $(\mathrm{p}=0.053)$ (19). Mukohara et al reported that sensitivity to NVP-AEW541 in MCF7 cells was due to high expression of both IGF1R and IRS1 (28).

Importantly, in the cell line model of acquired trastuzumab resistance, BT474/Tr, the triple combination appears to overcome trastuzumab resistance, and achieved greater than 90\% growth inhibition. Thus the triple combination may be beneficial for patients with trastuzumab-refractory HER2/ER/ IGF1R positive metastatic breast cancer. The triple targeting strategy may also represent a promising chemotherapy-free adjuvant treatment strategy for low risk HER2 positive breast cancer patients. It would be interesting to test this strategy in a chemotherapy-free study similar to the perioperative EPHOS-B study of trastuzumab and lapatinib (29).

To date clinical trials targeting IGF signaling, using TKIs or monoclonal antibodies targeting the receptor have produced disappointing results $(30,31)$ and those results combined with TKI-related toxicities have led to the termination of several IGF1R targeted therapies, including the two TKIs tested in this study. The lack of appropriate predictive biomarkers and the selection of patient subgroups most likely to respond to IGF1R inhibition is one of the major factors contributing to the failure of the clinical studies. Optimal combination strategies also need to be identified using preclinical studies. Thus targeting IGF1R in combination with hormone therapy in HER2/ER/ IGF1R positive breast cancer may be more successful than the previous IGF1R breast cancer clinical trials which were performed in ER positive breast cancer.

As previously mentioned IGF ligands may play a critical role in determining response/resistance to IGF1R inhibition $(32,33)$. Targeting IGF ligands may be a promising therapeutic approach to block IGF1R signaling and in addition to block IGF2 mediated insulin receptor signaling. Two monoclonal antibodies, BI 836845 and MEDI-573 which target IGF1 and IGF2, are currently in clinical development $(34,35)$. They have shown anti-proliferative activity in a range of cancer models and are currently in phase I/II studies in ER positive breast cancer.

In conclusion, our study provides significant evidence to suggest that targeting HER2, ER and IGF1R could have clinical benefit in the subgroup of HER2 positive patients whose tumors co-express ER and IGF1R. Evaluation of the dual (ER/IGF1R) and/or triple (HER2/ER/IGF1R) targeting combinations in preclinical in vivo models of breast cancer, in particular patient derived xenograft models, would be required to further evaluate the potential clinical benefit of this treatment strategy.

\section{Acknowledgements}

This study was supported by funding from the Irish Research Council (MMcD), the Health Research Board (CSA/2007/11), the Cancer Clinical Research Trust, Science Foundation Ireland through Molecular Therapeutics for Cancer, Ireland (08/SRC/B1410) and the Irish Cancer Society Collaborative Cancer Research Centre Breast-Predict (CCRC13GAL). 'The opinions, findings and conclusions or recommendations expressed in this material are those of the author(s) and do not necessarily reflect the views of the Irish Cancer Society'.

\section{References}

1. Nahta R and O'Regan RM: Therapeutic implications of estrogen receptor signaling in HER2-positive breast cancers. Breast Cancer Res Treat 135: 39-48, 2012.

2. Benz CC, Scott GK, Sarup JC, Johnson RM, Tripathy D, Coronado E, Shepard HM and Osborne CK: Estrogen-dependent, tamoxifen-resistant tumorigenic growth of MCF-7 cells transfected with HER2/neu. Breast Cancer Res Treat 24: 85-95, 1992.

3. Bhargava R, Dabbs DJ, Beriwal S, Yildiz IA, Badve P, Soran A, Johnson RR, Brufsky AM, Lembersky BC, McGuire KP, et al: Semiquantitative hormone receptor level influences response to trastuzumab-containing neoadjuvant chemotherapy in HER2positive breast cancer. Mod Pathol 24: 367-374, 2011. 
4. Vici P, Pizzuti L, Sperduti I, Frassoldati A, Natoli C, Gamucci T, Tomao S, Michelotti A, Moscetti L, Gori S, et al: 'Triple positive' early breast cancer: An observational multicenter retrospective analysis of outcome. Oncotarget 7: 17932-17944, 2016.

5. Kaufman B, Mackey JR, Clemens MR, Bapsy PP, Vaid A, Wardley A, Tjulandin S, Jahn M, Lehle M, Feyereislova A, et al: Trastuzumab plus anastrozole versus anastrozole alone for the treatment of postmenopausal women with human epidermal growth factor receptor 2-positive, hormone receptor-positive metastatic breast cancer: Results from the randomized phase III TAnDEM study. J Clin Oncol 27: 5529-5537, 2009.

6. Johnston S, Pippen J Jr, Pivot X, Lichinitser M, Sadeghi S, Dieras V, Gomez HL, Romieu G, Manikhas A, Kennedy MJ, et al: Lapatinib combined with letrozole versus letrozole and placebo as first-line therapy for postmenopausal hormone receptor-positive metastatic breast cancer. J Clin Oncol 27: 5538-5546, 2009.

7. Lee AV, Jackson JG, Gooch JL, Hilsenbeck SG, CoronadoHeinsohn E, Osborne CK and Yee D: Enhancement of insulin-like growth factor signaling in human breast cancer: Estrogen regulation of insulin receptor substrate-1 expression in vitro and in vivo. Mol Endocrinol 13: 787-796, 1999.

8. Molloy CA, May FEB and Westley BR: Insulin receptor substrate-1 expression is regulated by estrogen in the MCF-7 human breast cancer cell line. J Biol Chem 275: 12565-12571, 2000.

9. Hamelers IH and Steenbergh PH: Interactions between estrogen and insulin-like growth factor signaling pathways in human breast tumor cells. Endocr Relat Cancer 10: 331-345, 2003.

10. Casa AJ, Potter AS, Malik S, Lazard Z, Kuiatse I, Kim HT, Tsimelzon A, Creighton CJ, Hilsenbeck SG, Brown PH, et al: Estrogen and insulin-like growth factor-I (IGF-I) independently down-regulate critical repressors of breast cancer growth. Breast Cancer Res Treat 132: 61-73, 2012.

11. Ye JJ, Liang SJ, Guo N, Li SL, Wu AM, Giannini S, Sachdev D, Yee D, Brünner N, Ikle D, et al: Combined effects of tamoxifen and a chimeric humanized single chain antibody against the type I IGF receptor on breast tumor growth in vivo. Horm Metab Res 35: 836-842, 2003.

12. Lisztwan J, Pornon A, Chen B, Chen S and Evans DB: The aromatase inhibitor letrozole and inhibitors of insulin-like growth factor I receptor synergistically induce apoptosis in in vitro models of estrogen-dependent breast cancer. Breast Cancer Res 10: R56, 2008.

13. Chakraborty AK, Welsh A and Digiovanna MP: Co-targeting the insulin-like growth factor I receptor enhances growthinhibitory and pro-apoptotic effects of anti-estrogens in human breast cancer cell lines. Breast Cancer Res Treat 120: 327-335, 2010.

14. Hou X, Huang F, Macedo LF, Harrington SC, Reeves KA, Greer A, Finckenstein FG, Brodie A, Gottardis MM, Carboni JM, et al: Dual IGF-1R/InsR inhibitor BMS-754807 synergizes with hormonal agents in treatment of estrogen-dependent breast cancer. Cancer Res 71: 7597-7607, 2011.

15. Robertson JFR, Ferrero JM, Bourgeois H, Kennecke H, de Boer RH, Jacot W, McGreivy J, Suzuki S, Zhu M, McCaffery I, et al: Ganitumab with either exemestane or fulvestrant for postmenopausal women with advanced, hormone-receptor-positive breast cancer: A randomised, controlled, double-blind, phase 2 trial. Lancet Oncol 14: 228-235, 2013.

16. Lu Y, Zi X, Zhao Y, Mascarenhas D and Pollak M: Insulin-like growth factor-I receptor signaling and resistance to trastuzumab (Herceptin). J Natl Cancer Inst 93: 1852-1857, 2001

17. Nahta R, Yuan LXH, Zhang B, Kobayashi R and Esteva FJ: Insulin-like growth factor-I receptor/human epidermal growth factor receptor 2 heterodimerization contributes to trastuzumab resistance of breast cancer cells. Cancer Res 65: 11118-11128, 2005.

18. Hartog H, Van Der Graaf WTA, Boezen HM and Wesseling J: Treatment of breast cancer cells by IGF1R tyrosine kinase inhibitor combined with conventional systemic drugs. Anticancer Res 32: 1309-1318, 2012

19. Browne BC, Eustace AJ, Kennedy S, O'Brien NA, Pedersen K, McDermott MSJ, Larkin A, Ballot J, Mahgoub T, Sclafani F, et al: Evaluation of IGF1R and phosphorylated IGF1R as targets in HER2-positive breast cancer cell lines and tumours. Breast Cancer Res Treat 136: 717-727, 2012.
20. Haluska P, Bernath AM, Ballman KV, Dueck AC, Linden HM, Goetz MP, Northfelt DW, Hou X, Tenner KS, Tienchaiananda P, et al: Randomized phase II trial of capecitabine and lapatinib with or without cixutumumab in patients with HER2 breast cancer previously treated with trastuzumab and an anthracycline and/or a taxane: NCCTG N0733 (Alliance). J Clin Oncol 32 (Suppl 5): 632, 2014

21. Konecny GE, Pegram MD, Venkatesan N, Finn R, Yang G, Rahmeh M, Untch M, Rusnak DW, Spehar G, Mullin RJ, et al: Activity of the dual kinase inhibitor lapatinib (GW572016) against HER-2-overexpressing and trastuzumab-treated breast cancer cells. Cancer Res 66: 1630-1639, 2006.

22. Madden SF, Clarke C, Gaule P, Aherne ST, O'Donovan N, Clynes M, Crown J and Gallagher WM: BreastMark: An integrated approach to mining publicly available transcriptomic datasets relating to breast cancer outcome. Breast Cancer Res 15: R52, 2013.

23. Sorlie T, Tibshirani R, Parker J, Hastie T, Marron JS, Nobel A, Deng S, Johnsen H, Pesich R, Geisler S, et al: Repeated observation of breast tumor subtypes in independent gene expression data sets. Proc Natl Acad Sci USA 100: 8418-8423, 2003.

24. Dupont J and Le Roith D: Insulin-like growth factor 1 and oestradiol promote cell proliferation of MCF-7 breast cancer cells: New insights into their synergistic effects. Mol Pathol 54: 149-154, 2001.

25. García-Echeverría C, Pearson MA, Marti A, Meyer T, Mestan J, Zimmermann J, Gao J, Brueggen J, Capraro HG, Cozens R, et al: In vivo antitumor activity of NVP-AEW541-A novel, potent, and selective inhibitor of the IGF-IR kinase. Cancer Cell 5: 231-239, 2004.

26. Wittman M, Carboni J, Attar R, Balasubramanian B, Balimane P, Brassil P, Beaulieu F, Chang C, Clarke W, Dell J, et al: Discovery of a (1H-benzoimidazol-2-yl)-1H-pyridin-2-one (BMS-536924) inhibitor of insulin-like growth factor I receptor kinase with in vivo antitumor activity. J Med Chem 48: 5639-5643, 2005.

27. Ferguson RD, Gallagher EJ, Cohen D, Tobin-Hess A, Alikhani N, Novosyadlyy R, Haddad N, Yakar S and LeRoith D: Hyperinsulinemia promotes metastasis to the lung in a mouse model of Her2-mediated breast cancer. Endocr Relat Cancer 20: 391-401, 2013.

28. Mukohara T, Shimada H, Ogasawara N, Wanikawa R, Shimomura M, Nakatsura T, Ishii G, Park JO, Jänne PA, Saijo N et al: Sensitivity of breast cancer cell lines to the novel insulinlike growth factor-1 receptor (IGF-1R) inhibitor NVP-AEW541 is dependent on the level of IRS-1 expression. Cancer Lett 282: 14-24, 2009.

29. Bundred N, Cameron D, Armstrong A, Brunt A, Cramer A, Dodwell D, Evans A, Hanby A, Hartup S, Hong A, et al: Effects of perioperative lapatinib and trastuzumab, alone and in combination, in early HER $2^{+}$breast cancer - the UK EPHOS-B trial (CRUK/08/002). Eur J Cancer 57 (Suppl 2): LBA6, 2016.

30. Yee D: Insulin-like growth factor receptor inhibitors: Baby or the bathwater? J Natl Cancer Inst 104: 975-981, 2012.

31. Crudden C, Girnita A and Girnita L: Targeting the IGF-1R: The tale of the tortoise and the hare. Front Endocrinol (Lausanne) 6: $64,2015$.

32. Javle MM, Shroff RT, Varadhachary GR, Wolff RA, Fogelman DR, Bhosale P, Wang X, Kar SP, Overman MJ, Sathyanarayanan S, et al: Tumor IGF-1 expression as a predictive biomarker for IGF1R-directed therapy in advanced pancreatic cancer (APC) J Clin Oncol 30 (Suppl): 4054, 2012.

33. McCaffery I, Tudor Y, Deng H, Tang R, Suzuki S, Badola S, Kindler HL, Fuchs CS, Loh E, Patterson SD, et al: Putative predictive biomarkers of survival in patients with metastatic pancreatic adenocarcinoma treated with gemcitabine and ganitumab, an IGF1R inhibitor. Clin Cancer Res 19: 4282-4289, 2013.

34. Gao J, Chesebrough JW, Cartlidge SA, Ricketts SA, Incognito L, Veldman-Jones M, Blakey DC, Tabrizi M, Jallal B, Trail PA, et al: Dual IGF-I/II-neutralizing antibody MEDI-573 potently inhibits IGF signaling and tumor growth. Cancer Res 71: 1029-1040, 2011.

35. Friedbichler K, Hofmann MH, Kroez M, Ostermann E, Lamche HR, Koessl C, Borges E, Pollak MN, Adolf G and Adam PJ: Pharmacodynamic and antineoplastic activity of BI 836845, a fully human IGF ligand-neutralizing antibody, and mechanistic rationale for combination with rapamycin. Mol Cancer Ther 13: 399-409, 2014. 